\title{
Erratum
}

Bull Volcanol (1987) 49:765-775

\section{Variation in peperite textures associated with differing host-sediment properties}

\author{
Cathy J Busby-Spera and James DL White \\ Department of Geological Sciences, University of California, Santa Barbara, CA 93106, USA
}

Table 2 in the above article was misleading and unclear as printed. The corrected version is given below:

Table 2. Peperite characteristics observed at Punta China, Baja California, Mexico, with inferred processed of development. More detailed explanations in text

\begin{tabular}{|c|c|c|c|c|}
\hline \multirow[b]{2}{*}{ Host } & \multicolumn{2}{|c|}{ Fluidal peperites } & \multicolumn{2}{|c|}{ Blocky, hydroclastic peperites } \\
\hline & $\begin{array}{l}\text { unconsolidated } \\
\text { micrite, very fine } \\
\text { grained }\end{array}$ & $\begin{array}{l}\text { unconsolidated } \\
\text { micrite, very fine } \\
\text { grained }\end{array}$ & $\begin{array}{l}\text { lithic lapilli tuff brec- } \\
\text { cia, poorly sorted but } \\
\text { generally ash-poor }\end{array}$ & $\begin{array}{l}\text { lithic lapilli tuff brec- } \\
\text { cia, poorly sorted but } \\
\text { generally ash-poor }\end{array}$ \\
\hline Host permeability & very low (millidarcys?) & very low (millidarcys?) & $\begin{array}{l}\text { very high (tens of } \\
\text { darcys }^{a} \text { ) }\end{array}$ & $\begin{array}{l}\text { very high (tens of } \\
\text { darcys }^{a} \text { ) }\end{array}$ \\
\hline Host water content & $\begin{array}{l}\text { high }(40 \%) \text {, } \\
\text { diffuse }\end{array}$ & $\begin{array}{l}\text { high }(40 \%) \\
\text { diffuse }\end{array}$ & $\begin{array}{l}\text { high }(30 \%) \\
\text { localized }\end{array}$ & $\begin{array}{l}\text { high }(30 \%) \text {, } \\
\text { localized }\end{array}$ \\
\hline Morphology & Micro-Globular & Globular & Blocky & Dispersed \\
\hline $\begin{array}{l}\text { Peperite fragment } \\
\text { shape }\end{array}$ & $\begin{array}{l}\text { small (mm-scale) } \\
\text { micro-fluidal }\end{array}$ & $\begin{array}{l}\text { medium } \\
\text { (cm-dm scale) } \\
\text { macro-fluidal }\end{array}$ & $\begin{array}{l}\text { small (mm scale) } \\
\text { angular fragments }\end{array}$ & $\begin{array}{l}\text { large (dm-m scale) } \\
\text { angular fragments }\end{array}$ \\
\hline $\begin{array}{l}\text { Peperite fragment- } \\
\text { forming mechanism }\end{array}$ & $\begin{array}{l}\text { fluid } \\
\text { interface } \\
\text { instabilities }\end{array}$ & $\begin{array}{l}\text { fluid } \\
\text { interface } \\
\text { instabilities(?) }\end{array}$ & $\begin{array}{l}\text { dynamic stressing } \\
\text { granulation }\end{array}$ & $\begin{array}{l}\text { steam } \\
\text { explosions }\end{array}$ \\
\hline Fluidization pipes & $\begin{array}{l}\text { mm-scale } \\
\text { only }\end{array}$ & $\begin{array}{l}\mathrm{mm} \text {-scale } \\
\text { only }\end{array}$ & $\begin{array}{l}\text { meter-scale } \\
\text { pipes }\end{array}$ & $\begin{array}{l}\text { meter-scale } \\
\text { pipes }\end{array}$ \\
\hline Mixing mechanisms & $\begin{array}{l}\text { immiscibility, } \\
\text { fluidization, } \\
\text { "oscillation- } \\
\text { pumping" }\end{array}$ & $\begin{array}{l}\text { immiscibility, } \\
\text { fluidization, } \\
\text { fluid density } \\
\text { differences }\end{array}$ & $\begin{array}{l}\text { dynamic, } \\
\text { or unmixed }\end{array}$ & $\begin{array}{l}\text { steam } \\
\text { explosions, } \\
\text { fluidization }\end{array}$ \\
\hline
\end{tabular}

a Permeability estimate for lithic lapilli tuff breccia extrapolated from values for uncompacted modern sands (Pettijohn et al. 1972) 